\title{
Diagnóstico de la comunicación interna en el Hotel Comodoro
}

DOI: https://doi.org/10.33262/ap.v3i1.39

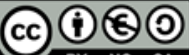

Diagnosis of internal communication at the Comodoro Hotel

Yulima Valdés Bencomo ${ }^{1}$, Yasser Vázquez Alfonso² \& Thai Dao Quang ${ }^{3}$

\begin{abstract}
.
The effectiveness of the hotel processes, as well as their quality depends in great measure of the participation of the workers in the actions and taking of decisions of the company. They also impact in great measure the organizational climate; where an atmosphere of comfortable and warm work and to maintain a good relationship between directive and subordinates impacts in the productivity of a company and consequently in their success in the market. In the previous mark, the communication plays a primordial paper in the establishment of the human relationships and of production; it is the key piece in the processes of an entity like factor transformer. In several investigations of the hotel context, the communication is an administration tool for the solution of problems. It is so the present investigation it proposes the diagnosis of the internal communication in the Hotel Commodore. The results of this investigation allow knowing the main characteristics of the Hotel Commodore and the perception of workers and directive on the internal communication in the hotel.
\end{abstract}

Keywords: Internal communication, communication actions, bidirectional communication.

\section{Resumen.}

\footnotetext{
${ }^{1}$ Universidad de La Habana, Facultad de Turismo. La Habana, Cuba. ybencomo@ftur.uh.cu

${ }^{2}$ Universidad de La Habana, Facultad de Turismo. La Habana, Cuba. yalfos1@gmail.com

${ }^{3}$ Universidad de La Habana, Facultad de Turismo. La Habana, Cuba. ybencomo@ftur.uh.cu
} 
La efectividad de los procesos hoteleros, así como su calidad dependen en gran medida de la participación de los trabajadores en las acciones y toma de decisiones de la empresa. También inciden en gran medida el clima organizacional; donde un ambiente de trabajo confortable y cálido y mantener una buena relación entre directivos y subordinados inciden en la productividad de una empresa y por consiguiente en su éxito en el mercado. En el marco anterior, la comunicación juega un papel primordial en el establecimiento de las relaciones humanas y de producción; además es la pieza clave en los procesos de una entidad como factor transformador. En varias investigaciones del contexto hotelero la comunicación es una herramienta de gestión para la solución de problemas. Es así que la presente investigación propone el diagnóstico de la comunicación interna en el Hotel Comodoro. Los resultados de esta investigación permiten conocer las principales características del Hotel Comodoro y la percepción de trabajadores y directivos sobre la comunicación interna en el hotel.

Palabras claves: Comunicación interna, acciones de comunicación, comunicación bidireccional.

\section{Introducción.}

El turismo es una actividad socio-económica que desde sus inicios ha contribuido al desarrollo de muchas naciones. En los últimos años el aporte de esta actividad trasciende a la generación de divisas, empleos, desarrollo de la infraestructura; así como la mantención y protección de recursos. En sentido general, si se observan cifras de la Organización Mundial del Turismo (OMT) se puede pensar el turismo como una actividad próspera, pues en el año 2018 se alcanzaron los 1.400 millones de arribos internacionales. Además, en cuanto a la generación de empleo se registró que más de 120 millones de empleos han sido generados por el sector (OMT, 2019).

Por otra parte, el turismo es una actividad económica muy sensible a las transformaciones de la sociedad. En las últimas dos décadas las tecnologías digitales han determinado el comportamiento de las personas: en las formas de viajar, preparar sus viajes e incluso disfrutar la experiencia en el destino. Desde la perspectiva del proveedor los cambios en los modelos de negocios, empleo de recursos y maneras de comunicarse con la demanda. Entonces, el esfuerzo por parte de los proveedores de adecuarse a los requerimientos de la demanda y destacarse sobre la competencia.

En Cuba, el turismo sigue siendo una de las principales fuentes de ingresos de divisas; además contribuye a la generación de empleos y potencia otros sectores de la economía como son la agricultura y la construcción. En los últimos años las cifras de los visitantes han ido favorablemente en ascenso. De acuerdo con los pronósticos oficiales en 2018 la isla recibió alrededor de cinco millones de vacacionistas extranjeros, lo que significó un crecimiento de más del seis por ciento en comparación con lo logrado en 2017 (Xinhuanet, 2018). 
En el contexto actual, el turismo atraviesa por una de las crisis más grandes que haya podido tener ante la pandemia causada por el virus SARS-COVID2. Las restricciones de confinamiento en todos los países con el fin de detener la propagación del virus han ocasionado que los arribos turísticos sean casi nulos en los destinos del mundo. Por otra parte, el turismo continúa siendo una fuente de ingresos importante para muchas comunidades vulnerables y algunas naciones se plantean alternativas para que esta actividad se recupere poco a poco. En el caso particular de $\mathrm{Cuba}$, actualmente se están creando las condiciones infraestructurales para cuando la apertura sea oportuna (Caraballoso, 2020); además se diseñó un Plan para la prevención y control de COVID-19. Por otra parte, muchas instalaciones turísticas cubanas acorde a sus características necesitan de planificar su apertura para que sea organizada y exitosa. En esta situación, la competencia será igual o más cruda que antes, y ya no solo basta con contar con una oferta de calidad y diferenciada; sino que sea segura. En este aspecto la comunicación juega un papel clave en la organización, pues es determinante en la forma en que las organizaciones se relacionan y se muestran hacia sus públicos.

La comunicación en las organizaciones turísticas es un instrumento de resolución de problemas. Bajo la idea anterior se parte de que, para mejorar el desempeño de las empresas en consecución con los objetivos establecidos y el grado de satisfacción de los clientes externos, es importante resaltar el papel de los empleados (Beprisma, 2016). He aquí una idea fundamental que hace reflexionar sobre el éxito del sector turístico en el país. Se considera que, en el punto de partida para alcanzar los logros propuestos, a nivel empresarial o sectorial, es la satisfacción y motivación de los empleados en el sector. Visto de esta forma, se debe prestar mayor atención al estado de satisfacción, motivación y liderazgo a través de la comunicación interna pues, constituye un aspecto fundamental dentro de todas las organizaciones.

En este sentido la comunicación interna es una de las principales herramientas con que cuentan las empresas para transmitir sus valores, visión y objetivos entre sus colaboradores. De ella depende en buena medida que el personal esté alineado con la estrategia corporativa y que los equipos sean lo suficientemente efectivos y productivos para alcanzar con éxito los beneficios de los negocios deseados. Por tanto, cuidar este canal de comunicación es esencial.

Gran parte de los problemas en las organizaciones están directamente relacionados con una insuficiente gestión de comunicación interna. No resolver los problemas a tiempo por falta de conocimiento, análisis, herramientas adecuadas o abordaje profesional compromete a la organización y acabará teniendo consecuencias negativas en sus resultados.

En el Hotel Comodoro el período comprendido de octubre a diciembre del presente año 2019, se pudo observar que los comensales que visitaban se sentían a gusto con los servicios y productos que se ofertaban de forma general; sin embargo, hacia lo interno de la instalación se notó cierto descontento en los empleados. A raíz de lo anterior surge una contradicción 
entre el prestigio ganado por el posicionamiento de la institución y la insuficiente comunicación interna de trabajadores y directivos, pues la presente investigación arrojo que en la institución no fluía la información, se evidencia vulnerabilidad al rumor, indiferencia recíproca, estancamiento, monotonía, falta de motivación en las actividades que se realizan, así como se comenzaba a percibiere éxodo de los trabajadores, evidenciándose que la situación anterior no era ajena para la alta dirección de la entidad, por lo que se requiere de un estudio que indague sobre cuáles son las principales causas que ocasionan la insatisfacción de los empleados y a partir de ello encontrar las soluciones pertinentes para mejorar dicha situación. Para ello se plantea como objetivo principal de la investigación: diagnosticar la comunicación interna del hotel Comodoro basado en la participación activa de trabajadores y directivos.

\section{Materiales y métodos.}

El Hotel Comodoro fue bautizado con ese nombre por el grado de Comodoro (con una estrella) (Commodore del francés Comandeur), Capitán de Navío, que en Inglaterra y otros países dirige una división de más de tres buques. Este grado existía en la Marina Cubana y el mismo estaba establecido en la Junta Directiva del Club. Se inauguró el 22 de noviembre de 1952. Se ubica en la zona residencial de Miramar se encuentra esta instalación privilegiada por una franja de playa privada. Ostenta una categoría de 4 estrellas y está administrado por el grupo hotelero cubano Cubanacán. La cartera de productos y servicios del Hotel Comodoro cuenta con cuatro líneas fundamentales: alojamiento, restauración, centro de negocios y recreación.

Para seguir y alcanzar los objetivos trazados en la planeación estratégica de la organización (al estar estos enfocados al ahorro y la gestión eficaz, unido a las limitaciones en los presupuestos de inversiones, renovación y reparaciones) la alta dirección del Hotel Comodoro ha adoptado una estrategia basada en los valores institucionales en correspondencia con la cultura organizacional propia.

La dirección de la entidad trabaja por promover una filosofía de Calidad y Atención al Cliente como un sistema de gestión eficaz con un enfoque estratégico que persigue la obtención de la calidad requerida y valorada por el cliente mediante un proceso de mejora continua, con énfasis en los procesos claves y no en las funciones, que involucre a los directivos y trabajadores con un fuerte compromiso para lograrla, sustentada por un programa de cambio estratégico definido, en el cual se trazaron las metas de la organización.

Perelló (2005), describe tres tipos de investigación científica en el sector turístico: la investigación histórica, la descriptiva y la experimental. La primera trata de la experiencia pasada, los objetivos de la investigación están enfocado en hacer una búsqueda crítica de la verdad que sustenta los acontecimientos del pasado. Por otro lado, la investigación descriptiva comprende la descripción, registro, análisis e interpretación de la naturaleza 
actual y la composición o procesos de los fenómenos. Además, trabaja sobre realidades de hecho para presentar interpretación correcta de la misma. Por último, está la investigación experimental que, a través de la manipulación de variables en condiciones controladas, se descubre de qué modo se comporta el fenómeno estudiado.

Evidentemente, esta investigación es de tipo descriptiva; pues a través de los resultados se describirá la situación actual en la comunicación interna, el estado de satisfacción de los trabajadores del Hotel Comodoro, así como permite un acercamiento panorámico al tema estudiado, todo ello bajo la observación del comportamiento en su contexto natural.

En cuanto a la información que se procesa la investigación puede ser cualitativa, cuantitativa o la combinación de ambas. La investigación cualitativa se utiliza cuando la información de que se dispone es pobre en datos, pero rica en descripciones de las variables, y no es fácilmente tratable con datos estadísticos. Por otro lado, cuantitativa basa su análisis en la información que proporciona los datos y fuentes de información (Peréz, 2001). En el caso de esta investigación es de tipo cuali-cuantitativa; pues se recurrirá al análisis y procesamiento de ambas informaciones.

Para el desarrollo de la investigación se consideró una como población el total de los trabajadores vinculados al Hotel Comodoro. La muestra se conformó por dos grupos de manera tal que permita evaluar el estado de satisfacción de los empleados y conocer cómo se caracteriza el proceso de comunicación interna.

En el caso de la evaluación del estado de satisfacción de los empleados, se procedió con un muestreo de la investigación de tipo no probabilístico intencional como plantea (Hernández et. al., 2006) "la elección de los elementos no depende de la probabilidad, sino de causas relacionadas con las características de la investigación o de quien hace la muestra". Visto esto, la muestra se concibe para 65 empleados.

Con respecto a conocer la situación de la comunicación interna se conformó una muestra de tipo intencional considerando los siguientes criterios:

- Directivos: jefes de departamento

- Empleados: representativo de cada departamento de forma aleatoria.

- Mínimo 5 años de trabajo en la organización.

En la investigación se ejecutaron diferentes etapas:

\section{Etapa 1: Acercamiento teórico.}

Para abordar este punto se tomó como referencia la clasificación que brinda Perelló (2005) acerca de las fuentes de procedencia de la información que se reflejan en la investigación, refriéndose a la interna como aquella que pertenece a la compañía objeto de estudio, la 
externa la que está fuera de esta, la primaria la captada únicamente para la investigación y como secundaria la que se encuentra a disposición del investigador en el momento de comenzar el estudio. En la presente investigación queda reflejado de la siguiente forma:

- Primaria/Interna: Criterios obtenidos a través de diferentes trabajadores de la entidad acerca del tema en cuestión, observación de la actividad y documentos específicos que describen el trabajo que se realiza acerca del tema de comunicación promocional en la empresa.

- Primaria /Externa: Consulta de documentos que brindaron datos referenciales acerca del tema en cuestión. Revisión de investigaciones anteriores relacionadas con el tema de investigación.

- Secundaria/Interna: Consulta de documentos que brindaron información secundaria acerca de la entidad y sus características, así como el funcionamiento de la actividad que se realiza en general a través de manuales, planes y diferentes materiales que se obtuvieron en las visitas realizadas.

- Secundaria/Externa: Documentos que tratan temas generales de interés acerca del sector turístico.

En la selección de los métodos y técnicas se tuvo en cuenta que la investigación es esencialmente de tipo descriptiva, cualitativa y cuantitativa. También fueron considerados los objetivos propuestos. En la investigación se emplearon tanto métodos teóricos como empíricos y estadísticos.

\section{Etapa 2: Organización y procesamiento de la información teórica}

\section{Métodos Teóricos.}

En la investigación se utilizó el método histórico-lógico para comprender la trayectoria y evolución del Hotel, tanto en el contexto nacional como el internacional. Por su parte, el método de análisis-síntesis facilitó la búsqueda y procesamiento de la información a través de la consulta bibliográfica, el estudio teórico y llegar a conclusiones. El método inductivodeductivo sirvió para determinar el estado actual del problema a resolver, la determinación de la hipótesis y el cuerpo de objetivos que permitió la concreción del proceso de investigación. Además, permitió determinar los factores claves de éxito de la instalación turística, los requerimientos de la organización del trabajo.

\section{Etapa 3: Elaboración del diagnóstico.}

\section{Métodos Empíricos:}


- Entrevista: Se pensó en recurrir a la entrevista semi-estructurada con el objetivo de conocer elementos de la comunicación interna desde la perspectiva de los gestores de la comunicación interna en el hotel. Sin embargo, dadas las condiciones de aislamiento no se procedió. Entonces se precedió a enviar un cuestionario a 5 directivos vía correo electrónico. El cuestionario para la entrevista se diseñó y utilizó con los siguientes objetivos: Identificar características del Hotel y determinar aspectos relacionados con el funcionamiento interno, incluyendo la comunicación interna.

- Encuesta: El cuestionario confeccionado para la encuesta se diseñó con el objetivo de identificar la la percepción que tienen los empleaos con respecto a la comunicación interna en la entidad.

El cuestionario está formado por una serie de preguntas tanto abiertas como cerradas, que buscan recopilar información entonto a las variables determinadas en la tabla 1.

Tabla 1. Variables presentes en la encuesta.

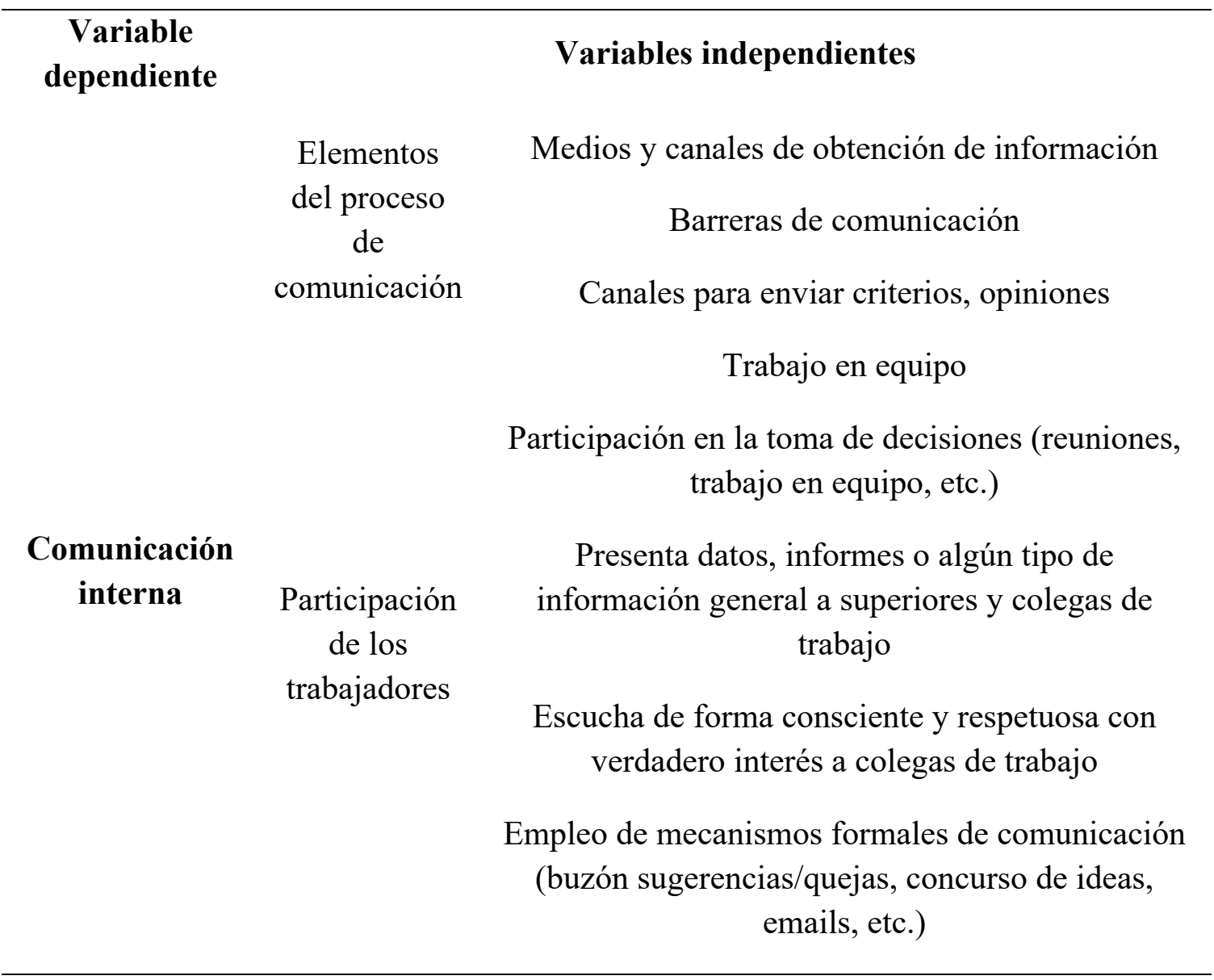


Contribución a la solución de conflictos interpersonales

Reconocimiento de su trabajo (parte de colegas de trabajo, jefes inmediatos y superiores)

Compensaciones

Motivación

Mecanismo corrección de errores (reprimendas, afectación de salario, críticas) Evaluación de desempeño (permite desarrollar habilidades, orientación positiva)

Nivel de seguridad

Fuente: Elaboración propia.

La observación es uno de los principales métodos empleados por los científicos, pues permite conocer la realidad mediante la sensopercepción directa de entes y procesos, para lo cual debe poseer algunas cualidades que le dan un carácter distintivo (Martínez \& Rodríguez, 2005). Es una percepción directa, atenta, racional, planificada de los fenómenos relacionados con los objetivos de la investigación, con vista a encontrar una explicación del fenómeno en estudio.

En esta investigación se aplicó la observación no participativa y abierta, pues las actividades observadas fueron de conocimiento general. Se realizó de forma semiestructurada.

La observación se realizó con los siguientes propósitos:

- Apreciar el estado de las áreas de Hotel

- Valorar la actitud de los encuestados en el proceso de contestación.

\section{Análisis de los resultados.}

- Estadística descriptiva: Se concibió para procesar las encuestas a través de gráficos, tablas y estadígrafos.

- Estadístico Multivariada. Se concibió para el procesamiento de las múltiples variables de la encuesta a través del Análisis de Correspondencia Múltiple y la aplicación del software MICMAC para la identificación de variables claves y su nivel de relaciones.

El diagnóstico de comunicación interna permite conocer las necesidades comunicativas y de información del público interno; además posibilita que el sistema de comunicación sea eficaz. 
ara el desarrollo de esta investigación, se asume el modelo de diagnóstico de la comunicación interna propuesto por la especialista cubana (Martinez, 2009), estudiosa del tema, con amplia experiencia práctica en instituciones de la Mayor de las Antillas, y que aparecen en el libro, de la editorial Logos de la Asociación Cubana de Comunicadores Sociales, aunque seleccionamos de esta autora los pasos necesarios según el interés de la investigación, por cuanto en la empresa ya se había realizado un diagnóstico de la imagen de esta organización.

Los aspectos a tener en cuenta en el diagnóstico son:

1. Aspectos de carácter general u orientativo sobre la organización orientada:

- Nombre, razón social, finalidad.

- Nivel de conocimiento sobre aspectos relacionados con la comunicación y manejo de los términos.

2. Aspectos de carácter estratégico (son aquellos que van a definir el eje desde el cual deben partir las acciones de comunicación):

- Definir si existe un planteamiento estratégico de la institución.

- Tener formulada su visión estratégica y los objetivos que se derivan de ella Evaluar si se corresponde con la realidad organizacional y son alcanzables estos planteamientos.

3. Aspectos de carácter funcional:

- Como está organizada estructural y funcionalmente la organización para el desarrollo de la tarea y el cumplimiento de sus objetivos

4. Aspectos propiamente de la actividad de comunicación:

- Existencia de un reconocimiento de los públicos que interactúan con la organización. Ello permite la definición posterior de un mapa de los públicos de la institución.

5. Aspectos de contenido de comunicación:

- Existencia de planteamientos conceptuales de comunicación.

- Existencia de definición de mensajes para los diversos públicos con los que opera la institución y que apoyen las metas organizacionales.

\section{Resultados y discusión.}


El proceso de comunicación esta mayor mente enfocado a la comunicación externa, para la que se disponen manuales y documentos aportados por el grupo hotelero (Manual de Comunicación y Relaciones Públicas). Se puede decir que estos objetivos presentan un enfoque comercial con el propósito de incrementar la imagen y la identidad corporativa, en resumen, promover los servicios y productos que oferta la organización a través de la aceptación de sus públicos.

En la figura 1 se muestra los valores de las categorías más frecuenta en los criterios de los trabajadores sobre la comunicación interna del hotel Comodoro. Se observa que el 91\% de los encuestados conocen la misión, visión y valores compartidos del hotel(PCMVVaCH), el $60 \%$ reconoce que los portavoces de información son la gerencia y el jefe inmediato(PPRI), el 70\% comenta que los principales canales de información que utiliza para informarse son reuniones informativas, teléfonos y colegas(CCII), los principales propósitos por los que se comunica es para precisar información sobre lo que deben hacer y qué resultados se esperan e informar sobre objetivos y planes de trabajo representando el 65\%( PPCS), los principales espacios en donde se puede emitir los criterios son en reuniones sindicales, entrevista y encuestas(EECIO), el 75\% opina que comparte información con los altos mandos(CQCIP), el $63 \%$ plantea que en las reuniones de trabajo toman nota de los comentarios que se realizan(ELRT), el 55\% comentan que se consideran sus criterios y opiniones en la toma de decisión(CCOSTS), el 70\% opina que desde su puesto de trabajo contribuye al desarrollo del hotel(CUSPTCH), entre los elementos que entorpecen la comunicación se encuentran las emociones como (frustración, temor, desconocimiento) y el Rumor/ incertidumbre(EECIC), las medidas correctivas más empleadas por el hotel según la experiencia de los encuestados son: destitución de cargos, críticas y señalamientos en expedientes representando 74\% (MCMEE),el 71\% considera que se trabaja en equipo (STE) y los principales problemas que afronta la comunicación en el trabajo en equipo son: ironía e indiferencia o individualismo (PPACTE).

Figura 1. Valores más frecuentes en los criterios de los encuestados.

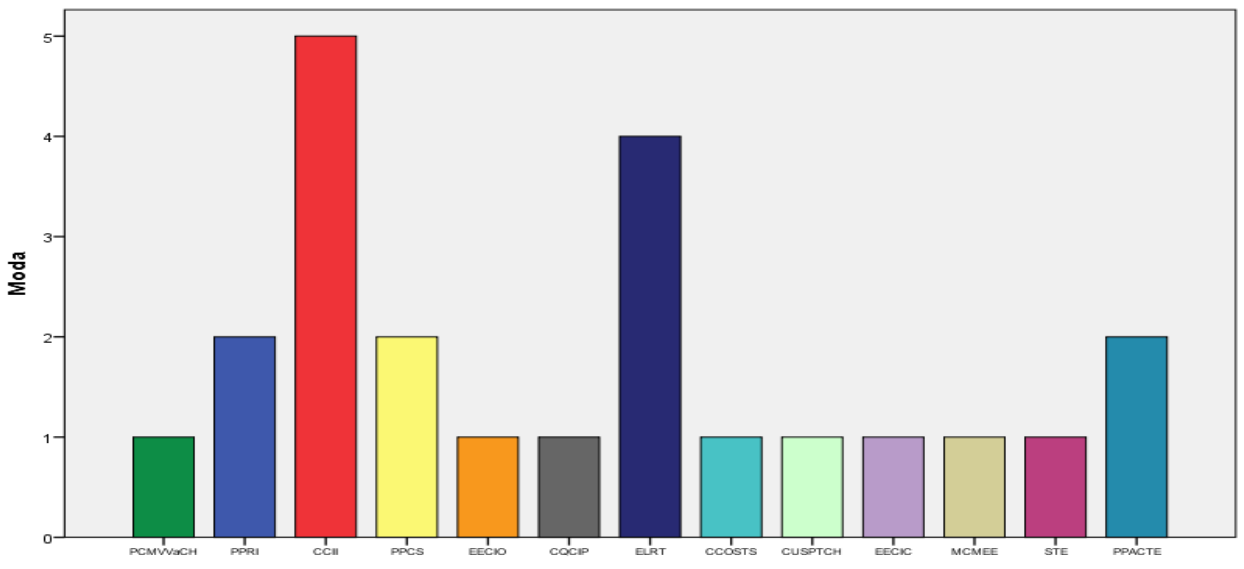

Fuente: Elaboración propia. 
Para un análisis más integral se realiza el estudio multivariable que se muestra en la figura 2 , donde a través del gráfico biplot se muestra que las variables que más peso tienen en la opinión de los trabajadores sobre la comunicación interna son: elementos entorpecen la comunicación interna en su centro (EECIC), principales propósitos por los que se comunica (PPCS), principales problemas que afronta la comunicación en el trabajo en equipo (PPACTE) y los canales de comunicación interna que usted se informa (CCII). El valor de Alfa de Cronbach es de 0,951 y está dentro de la fiabilidad ya que es mayor que 0,7 según (Hair et.al.;1999).

Figura 2. Gráfico biplot del peso de las variables.

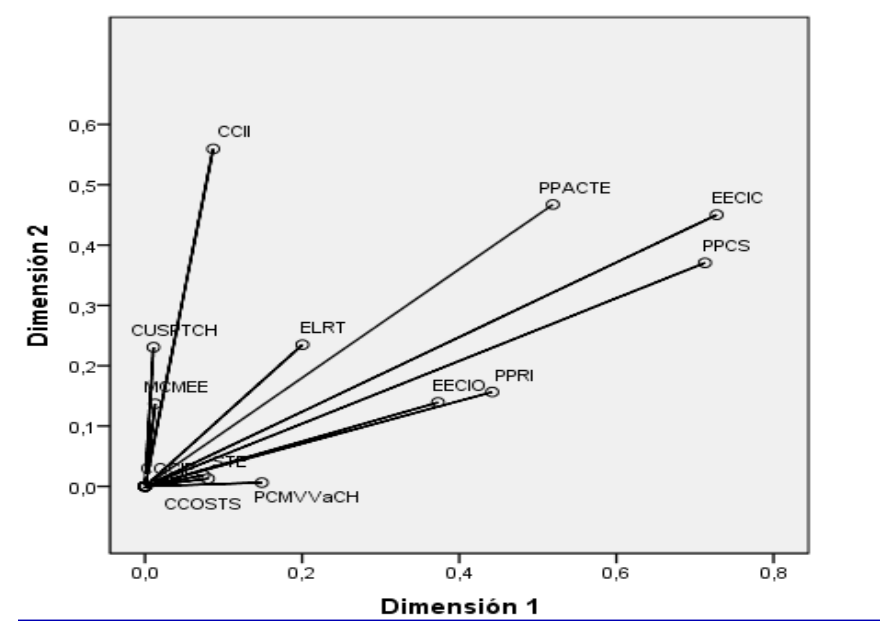

Fuente: Elaboración propia.

Con el objetivo de describir el comportamiento de las diferentes variables utilizadas en la encuesta, se aplica un análisis con el software MICMAC para identificar las variables influyentes y dependientes en el estudio. En la figura 3 se muestra el gráfico del plano de influencia de las variables analizadas y se observa que CQCIP(Con quien comparte información principalmente), CCII (canales de comunicación interna que usted se informa), MCMEE(medidas correctivas más empleadas por la entidad), CCOSTS (Considera los criterios y opiniones en la toma de decisiones) y CUSPTCH (Considera usted que desde su puesto de trabajo contribuye al desarrollo del Hotel), STE(Se trabaja en equipo), ELRT (En las reuniones de trabajo usted) y PCMVVaCH (percepción del conocimiento de la misión, visión y valores compartidos del hotel), se clasifican como variables autónomas ya que se ubican cerca del origen y se distinguen por ser poco dependientes, las variables elementos entorpecen la comunicación interna en su centro (EECIC), principales propósitos por los que se comunica (PPCS) y PPRI (principales portavoces por los que recibe información), se corresponden con las variables claves que se encuentran en la zona superior derecha y son muy dependientes. Las variables EECIO (espacios puede emitir su criterio, ideas opiniones), y PPACTE (principales problemas 
que afronta la comunicación en el trabajo en equipo), se identifican como variables reguladoras ya que se sitúan en la zona central y se convierten en elementos fundamentales para alcanzar el cumplimiento de las variables claves.

Figura 3. Plano de influencia y dependencia de las variables de la encuesta.

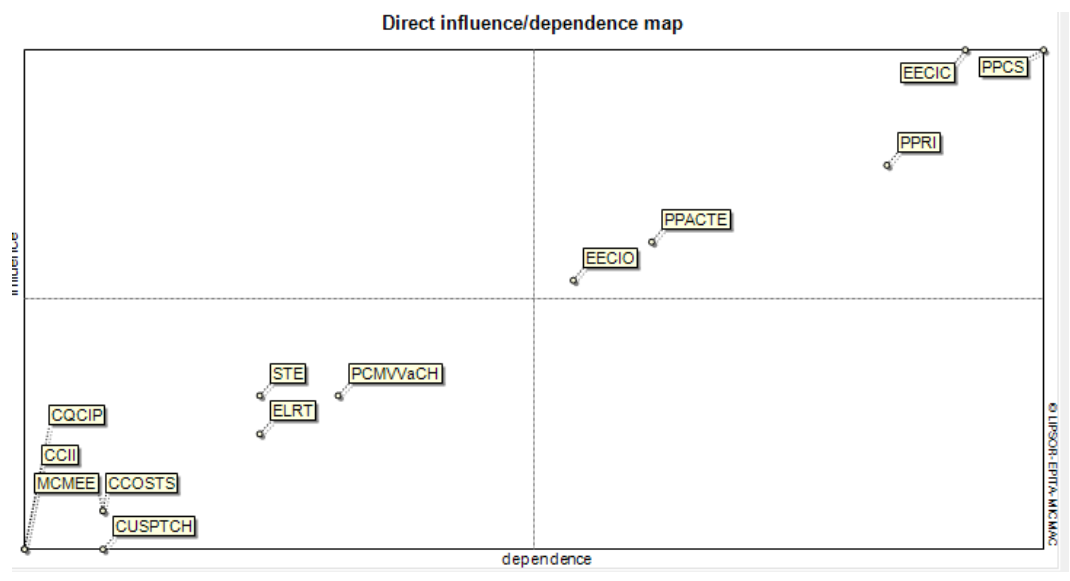

Fuente: Elaboración propia.

Otro resultado en la investigación fue la realización del gráfico de influencia directa que se detalla en la figura 4, donde aparecen las diferentes clasificaciones de las relaciones entre las variables de la encuesta. La relación más fuerte se establece entre elementos entorpecen la comunicación interna en su centro (EECIC) y los principales propósitos por los que se comunica (PPCS).

Figura 4. Gráfico de influencia directa

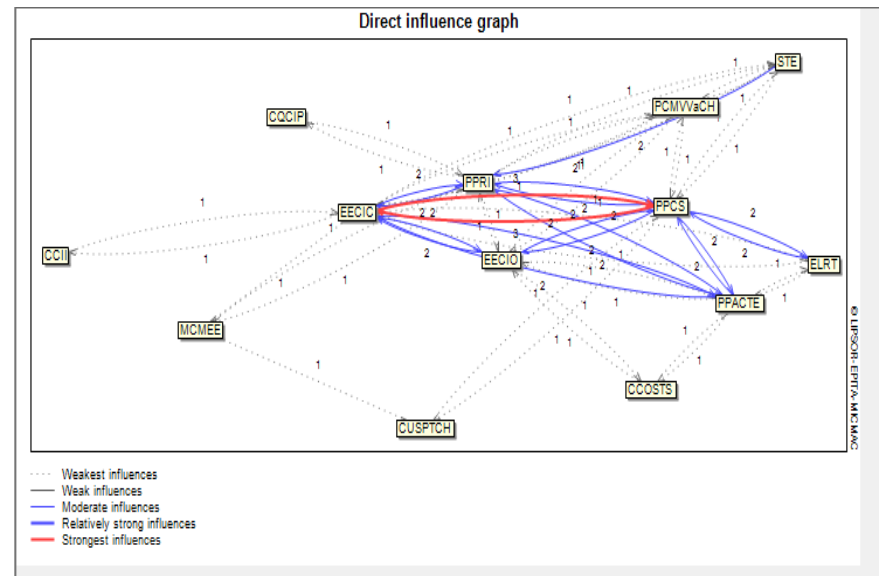

Fuente: Elaboración conjunta. 
Algunos de estos resultados coinciden con los estudios realizados sobre las barreras en la comunicación de las organizaciones por los autores Moreno (2009) y Montero (2017) que plantean los diferentes tipos de barreras como son: Psicológicas-Emocionales, Físicas y Semánticas. En la encuesta las que más se destacan es la semántica reflejada por la incertidumbre que refiere la poca claridad en qué es lo que se quiere lograr con el mensaje y cómo hacerlo, tiene un efecto psicológico en los empleados de la empresa puede evidenciarse en varias formas según (Martin, 2017) como con ansiedad o incluso pánico, el rumor es considerado como un tipo de comunicación informal (GALEON, 2006); puede ser negativo para la empresa si es que crea un ambiente de tensión, expectativa y desasosiego entre el personal.

\section{Conclusiones.}

- La comunicación en el hotel Comodoro tiende a fluir de forma descendente y puede tener un carácter bidireccional asimétrico; teniendo en cuenta el carácter del propósito por el cual comunican los directivos y la naturaleza del mismo. Se puede deducir limitaciones en la participación de los empleados; así como retroalimentación.

- El diagnóstico realizado evidencia que los directivos del hotel deben promover la comunicación interna y elevar o mantener estados satisfactorios en los empleados hacia la empresa; y redireccionar el flujo de la comunicación interna hacia un plano bidireccional simétrico, debe formar a los directivos y trabajadores en materias de de comunicación interna y ampliar los canales de comunicación empleados en la organización.

\section{Referencias bibliográficas.}

Beprisma (2016). 10 formas de mejorar la comunicación interna. Disponible en: https://beprisma.com/10-formas-mejorar-la-comunicacion-interna/

Caraballoso, E (2020). Pasos y claves de reapertura del turismo cubano. Disponible en:https://oncubanews.com/cuba/economía/turismo/pasos-y-claves-de-la-reaperturadel-turismo-cubano/amp/.

Galeon (2006). La Comunicación en la empresa. Disponible en: http://www.comunicacionempresa.galeon.com/productos2280371.html

Hair, J. F.; Anderson, R. E.; Tatham, R. L.; lack, W. C (1999). Análisis Multivariante. Practice Hall Iberia. Madrid. España. 799p.

Hernández, R., Fernández, C., \& Baptista, P. (2006). Metodología de la Investigación. México D. F.: McGraw-Hill Interamericana. 
Martin, D. (2017). ¿Incertidumbre en la empresa? 8 claves para ganar tranquilidad. Disponible en: https://www.estrategiapractica.com/como-trabajar-conincertidumbre/

Martínez, R., \& Rodríguez, E. (2005). Manual de Metodología de la Investigación Científica. Editorial UH. La Habana. p.34.

Martínez, Y. (2009). Hablemos de Comunicación. Logos de la Asociación Cubana de Comunicadores Sociales.

Montero, M. (2017). Herramientas de comunicación interna. Disponible en: https://www.emprendepyme.net/herramientas-de-comunicacion-interna.html

Moreno, L. Á. (2009). Comunicación Efectiva para el logro de una visión compartida. CULCyT (32), 5-19.

Perelló, J. (2005). Introducción a la investigación de mercados turísticos. La Habana.

Pérez, A. S. (2001). Apuntes de Metodología de la Investigación en Turismo

Xinhuanet. (2018). El turismo en Cuba: su importancia y buenos pronósticos. Disponible en: http://spanish.xinhuanet.com/2018-01/03/c_136868599.htm

OMT (2019). WNWTO Tourism Highlights 2019 Edition.

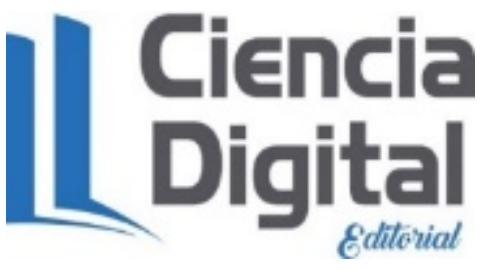




\section{Para citar el artículo indexado}

Valdés Bencomo, Y., Vázquez Alfonso, Y., \& Dao Quang, T. (2020). Diagnóstico de la comunicación interna en el Hotel Comodoro. AlfaPublicaciones, 3(1), 51-65. https://doi.org/10.33262/ap.v3i1.39

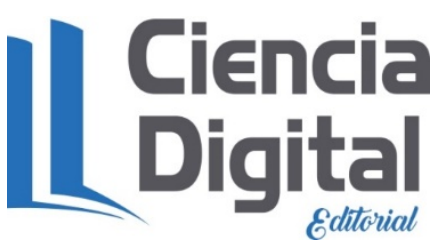

El artículo que se publica es de exclusiva responsabilidad de los autores y no necesariamente reflejan el pensamiento de la Revista Alpha Publicaciones.

El artículo queda en propiedad de la revista y, por tanto, su publicación parcial y/o total en otro medio tiene que ser autorizado por el director de la Revista Alpha Publicaciones.
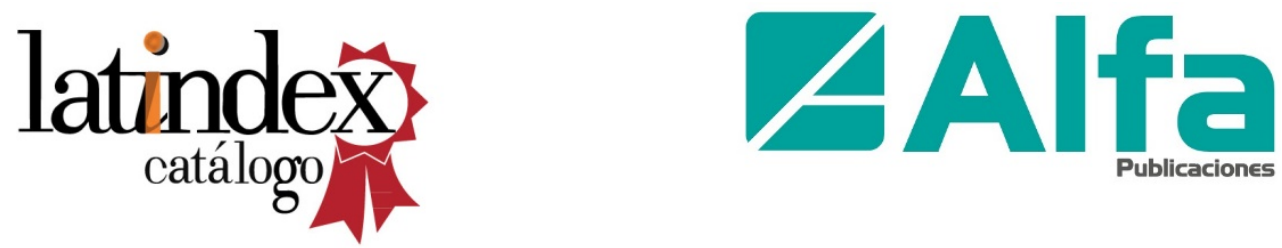